\title{
ON CLASSICAL QUOTIENTS OF POLYNOMIAL IDENTITY RINGS WITH INVOLUTION
}

\author{
LOUIS HALLE ROWEN ${ }^{1}$
}

\begin{abstract}
Let $(R, *)$ denote a ring $R$ with involution (*), where "involution" means "anti-automorphism of order $\leqq$ two". We can specialize many ring-theoretical concepts to rings with involution; in particular an ideal of $(R, *)$ is an ideal of $R$ stable under $(*)$, and the center of $(R, *)$ is the set of central elements of $R$ which are fixed under $(*)$. Then we say $(R, *)$ is prime when the product of any two nonzero ideals of $(R, *)$ is nonzero; similarly $(R, *)$ is semiprime when any power of a nonzero ideal of $(R, *)$ is nonzero. The main result of this paper is a strong analogue to Posner's theorem [5], namely that any prime $(R, *)$ with polynomial identity has a ring of quotients $R_{T}$, formed merely by adjoining inverses of nonzero elements of the center of $(R, *)$. This quotient ring $\left(R_{T}, *\right)$ is simple and finite dimensional over its center. An extension of these results to semiprime Goldie rings with polynomial identity is given.
\end{abstract}

1. Facts about arbitrary rings with involution. Throughout this paper, $R$ will denote a ring with 1 . Actually, it is easy to show that all that is needed in the sequel is for the center $C$ to contain a regular element. It happens that in light of [6, Theorem 2] this is always the case for $R$ prime with polynomial identity (and, more generally, for $R$ semiprime Goldie with polynomial identity) but we will not worry about this question here. Let us start with some elementary facts about arbitrary rings with 1 .

Lemma 1. Let $R$ be a ring (with 1 and center $C$ ). Let $T$ be any multiplicatively closed subset of regular elements (i.e. for $c \in T, r \in R, c r=0 \Rightarrow r=0$ ) of $C$ such that $1 \in T$. Define $R_{T}=\left\{r c^{-1}, r \in R, c \in T\right.$, such that $r_{1} c_{1}^{-1}=$ $r_{2} c_{2}^{-1}$ iff $\left.r_{1} c_{2}=r_{2} c_{1}\right\} . R_{T}$ is then a ring endowed with the following operations: $r_{1} c_{1}^{-1}+r_{2} c_{2}^{-1}=\left(r_{1} c_{2}+r_{2} c_{1}\right)\left(c_{1} c_{2}\right)^{-1}$ and $\left(r_{1} c_{1}^{-1}\right)\left(r_{2} c_{2}^{-1}\right)=\left(r_{1} r_{2}\right)\left(c_{1} c_{2}\right)^{-1}$, for $r_{1}, r_{2} \in R, c_{1}, c_{2} \in T . R \rightarrow R_{T}$ via $r \mapsto r 1^{-1}$, and $\operatorname{cent}\left(R_{T}\right)=C_{T}$.

Received by the editors October 6, 1972.

AMS (MOS) subject classifications (1970). Primary 16A08, 16A12, 16A38.

Key words and phrases. Classical ring of quotients, Goldie ring, involution, polynomial identity, prime, semiprime, simple, center, central quotients.

${ }^{1}$ The author is a doctoral student at Yale University, working under the guidance of Nathan Jacobson. This work was supported by a National Science Foundation fellowship and is part of the author's forthcoming dissertation.

(c) American Mathematical Society 1973 
Proof. It is clear that the given operations are well defined, making $R_{T}$ a ring, and the map given from $R$ to $R_{T}$ is an embedding. $C_{T} \subseteq \operatorname{cent}\left(R_{T}\right)$ is trivial; to show that $C_{T} \supseteq \operatorname{cent}\left(R_{T}\right)$, let $r c^{-1} \in \operatorname{cent}\left(R_{T}\right)$ for some $r \in R$, $c \in T$. Then for any $r_{1} \in R,\left(r c^{-1}\right)\left(r_{1} 1^{-1}\right)-\left(r_{1} 1^{-1}\right)\left(r c^{-1}\right)=0$, so by definition $\left(r r_{1}-r_{1} r\right) c=0$. Then $r r_{1}-r_{1} r=0$, so $r \in C$, which proves $C_{T} \supseteq \operatorname{cent}\left(R_{T}\right)$, so $C_{T}=\operatorname{cent}\left(R_{T}\right)$. Q.E.D.

Noting that the set of all regular elements of $C$ is multiplicative, we could let $T$ be this set in Lemma 1. In this case we shall call $R_{T}$ the ring of central quotients of $R . R_{T}$ is clearly universal in the following sense, for any $T$ as in Lemma 1.

Let $S$ be another ring (with 1), and let $\alpha: R \hookrightarrow S$ be a ring monomorphism such that $\alpha(c)$ is invertible in $S$ for all $c \in T$. Then, viewing $R$ as a subring of $R_{T}$, we have a unique ring monomorphism $\beta: R_{T} \rightarrow S$ extending $\alpha$. In particular, if $R$ has a (classical) ring of quotients $S$ [3, p. 261], then $R_{T} \subseteq S$.

We shall now develop the analogous situation for rings with involution $(R, *)$. The center $C_{0}$ of $(R, *)$ is defined as $\left\{c \in C\right.$ such that $\left.c^{*}=c\right\}$. $1^{*}=1$, so $1 \in C_{0}$.

LemMa 2. Let $(R, *)$ be a ring with involution (with 1 and center $C_{0}$ ). Let $T_{0}$ be any multiplicatively closed subset of regular elements of $C_{0}$, with $1 \in T_{0}$. Then $R_{T_{0}}$ has an involution which we shall also call (*), given by $\left(r c^{-1}\right)^{*}=r^{*} c^{-1}$ for $r \in R, c \in T_{0}$. The embedding $R \rightarrow R_{T_{0}}$ respects involution, and $\operatorname{cent}\left(R_{T_{0}}, *\right)=\left(C_{0}\right)_{T_{0}}$.

Proof. We observe that (*) is well defined on $R_{T_{0}}$, for if $r_{1} c_{1}^{-1}=r_{2} c_{2}^{-1}$, then $r_{1} c_{2}=r_{2} c_{1}$, so $r_{1}^{*} c_{2}=r_{2}^{*} c_{1}$, which implies $r_{1}^{*} c_{1}^{-1}=r_{2}^{*} c_{2}^{-1}$. It is likewise easy to see that (*) is indeed an involution on $R_{T_{0}}$. Since $r^{*} 1^{-1}=\left(r 1^{-1}\right)^{*}$, the embedding $R \rightarrow R_{T_{0}}$ does respect involution, and the last assertion follows immediately from Lemma 1 . Q.E.D.

Let us call (*) of the first kind on $R$ if $C=C_{0}$, and of the second kind on $R$ otherwise $\left(C \neq C_{0}\right)$. Then we observe that $(*)$ is of the same kind on $R$ as on $R_{T_{0}}$.

If $T_{0}=\left\{\right.$ all regular elements of $\left.C_{0}\right\}$ then we shall call $\left(R_{T_{0}}, *\right)$ the ring of central quotients of $(R, *)$, noting that $T_{0}$ is multiplicative.

Lemma 3. For $(R, *)$ a ring with involution, let $T=\{$ regular elements of $C\}$ and $T_{0}=\left\{\right.$ regular elements of $\left.C_{0}\right\}$. Then $R_{T_{0}}=R_{T}$; in other words, the ring of central quotients of $(R, *)$, considered without the involution, is the same as the ring of central quotients of $R$.

Proof. Since $T_{0}$ is a multiplicative set of regular elements of $C$, there is a canonical embedding $R_{T_{0}} \rightarrow R_{T}$ from considerations of universality, 
given by $r c_{0}^{-1} \mapsto r c_{0}^{-1}$ for $r \in R, c_{0} \in T_{0}$. But this map is onto, because for all $c \in T, c c^{*} \in T_{0}$, so that $r c^{-1}=r c^{*}\left(c c^{*}\right)^{-1} \in R_{T_{0}}$. So $R_{T_{0}}=R_{T}$. Q.E.D.

Having developed a suitable theory of central localization for rings with involution, we change direction somewhat to define more ring-theoretic structures on rings with involution.

Let $A$ be a subset of $R$. Then $A$ is an ideal of $(R, *)$ if $A$ is an ideal (2-sided) of $R$ and $A^{*}=A$, as in [4, p. 13].

In a manner analogous to [5], we make the following definitions:

$(R, *)$ is simple if the only ideals of $(R, *)$ are 0 and $R$.

$(R, *)$ is prime if the product of nonzero ideals of $(R, *)$ is always nonzero.

$(R, *)$ is semiprime if all powers of all nonzero ideals of $(R, *)$ are nonzero.

If $A$ is an ideal of $(R, *)$, then $R / A$ has an involution which we shall also call (*), given by $(r+A)^{*}=r^{*}+A$. Then we say $A$ is (maximal, prime, semiprime) if $(R / A, *)$ is (simple, prime, semiprime). Finally we say $(R, *)$ is semisimple if 0 is the intersection of maximal ideals of $(R, *)$.

Martindale showed that $(R, *)$ is semiprime if and only if $R$ is semiprime, using structure theory of semiprime rings [5, p. 193]. We prove this fact directly.

Clearly $R$ semiprime implies $(R, *)$ is semiprime, so let us prove the converse and assume $(R, *)$ is semiprime. Let $A$ be any ideal of $R$ such that $A^{2}=0$. Then $A A^{*}$ is an ideal of $(R, *)$ such that $\left(A A^{*}\right)\left(A A^{*}\right) \subseteq A^{2}=0$, so $A A^{*}=0$. Similarly $A^{*} A=0$, and $\left(A^{*}\right)^{2}=\left(A^{2}\right)^{*}=0$. Thus, $\left(A+A^{*}\right)^{2}=0$, so $A+A^{*}=0$ since $\left(A+A^{*}\right)$ is an ideal of $(R, *)$. But then $A=0$, so $R$ is indeed semiprime.

We will also need the observation that if $(R, *)$ is prime, then the nonzero elements of its center $C_{0}$ are regular. For suppose $c \in C_{0}$ and $r c=0$. Then $r^{*} c=r^{*} c^{*}=(c r)^{*}=(r c)^{*}=0$; since $c$ is central we have $\operatorname{Ann}_{R}(c)=$ $\{r \in R$ such that $r c=0\}$ is an ideal of $(R, *)$. Likewise $c R$ is an ideal of $(R, *)$, and $\left(\operatorname{Ann}_{R}(c)\right)(c R)=0$. Since $(R, *)$ is prime we conclude that $\mathrm{Ann}_{R}(c)=0$ or $c=0$, which proves our contention. In particular, if $(R, *)$ is prime then $C_{0}$ is an integral domain.

2. Polynomial identities. We still assume that $(R, *)$ is a ring with involution, and with 1 . Let $F$ be the free noncommutative ring $Z\left\{X_{11}, X_{12}, \cdots, X_{i 1}, X_{i 2}, \cdots\right\}$, where $Z$ is the ring of integers. Let us define an involution (*) on $F$ by $\alpha^{*}=\alpha$ for $\alpha \in Z, X_{i 1}^{*}=X_{i 2}, X_{i 2}^{*}=X_{i 1}$. Then let us write $X_{i}=X_{i 1}, X_{i}^{*}=X_{i 2}$, so $F=Z\left\{X_{1}, X_{1}^{*}, \cdots, X_{i}, X_{i}^{*}, \cdots\right\}$. We say $f \neq 0$ in $F$ is a polynomial identity of $(R, *)$ if $f$ is in the kernel of all homomorphisms of $(F, *)$ to $(R, *)$, where a homomorphism of $(F, *)$ to $(R, *)$ is defined as a homomorphism of $F$ to $R$ preserving the involution. Note 
that our definition of polynomial identity of $(R, *)$ is essentially a special case of Amitsur's treatment in [2, p. 64]. Incidentally, $(F, *)$ is a free ring with involution, in the sense that given any $(R, *)$ and $a_{i} \in R, i=1,2, \cdots$, there exists a unique homomorphism of $(F, *)$ to $(R, *)$ sending $X_{i}$ to $a_{i}$, $i=1,2, \cdots$.

Amitsur shows in [2] that with mild conditions on $f$ (one of the coefficients is \pm 1$), R$ satisfies a polynomial identity (in the usual sense). (This result generalizes work by Herstein and Martindale. Herstein showed that if $R$ is simple and if some nonvanishing polynomial vanishes for all substitutions of symmetric elements in $R$, then $R$ has a polynomial identity; Martindale extended Herstein's theorem for $R$ semiprime.) If $(R, *)$ is semiprime then since $R$ is also semiprime this polynomial identity may in fact be assumed to be a standard identity, which is multilinear. For a discussion on how to define polynomial identities on semiprime rings, see [1, p. 484].

Now let $R$ be a ring with homogeneous polynomial identity $f[3$, p. 224]. Then for any multiplicative set $T$ of regular central elements, $R_{T}$ also satisfies the polynomial identity $f$, because if $r_{i} \in R, c_{i} \in T$ then $f\left(r_{1} c_{1}^{-1}, \cdots, r_{m} c_{m}^{-1}\right)=f\left(r_{1}, \cdots, r_{m}\right) c^{-1}=0$ where $c$ is the product of each $c_{i}$ raised to the degree of the $i$ th variable of $f$. Since $R \rightarrow R_{T}$, it follows that $R$ and $R_{T}$ satisfy the same homogeneous polynomial identities. Now if $(R, *)$ satisfies a polynomial identity, then $R$ satisfies a polynomial identity, so $R$ satisfies a multilinear polynomial identity, which implies that $R_{T}$ satisfies the same multilinear identity. In particular, if $T=C_{0}=$ center of $(R, *)$, we see that the ring of central quotients of $(R, *)$ also satisfies a polynomial identity. Actually, one could define homogeneous identities of $(R, *)$ along the lines of [2] to show that for any multiplicative subset $T$ of $C_{c}$ with $1 \in T,(R, *)$ and $\left(R_{T}, *\right)$ satisfy the same homogeneous identities. Namely, let $f \in(F, *)$, and suppose $f=\sum f_{r}\left(X_{1}, X_{1}^{*}, \cdots, X_{m_{r}}, X_{m_{r}}^{*}\right)$ where each $f_{r}$ is a monomial. The degree of the ith indeterminate of $f_{r}$ is the sum of the degrees of $X_{i}$ and of $X_{i}^{*}$ in $f_{r}$. If this degree is independent of $r$ for each indeterminate then $f$ is homogeneous. Then one gets the desired result (that $f$ is a homogeneous polynomial identity for $\left(R_{T}, *\right)$ if and only if $f$ is a homogeneous polynomial identity for $(R, *))$ in the same way as discussed above.

Now we state and prove the analogue of [6, Theorem 2] for rings with involution.

Theorem 1. Let $(R, *)$ be semiprime with center $C_{0}$. Suppose $(R, *)$ satisfies a polynomial identity. Then for any nonzero ideal $A$ of $(R, *)$, $A \cap C_{0} \neq 0$.

Proof. We have already seen that $A$ is an ideal of $R$, which is semiprime with polynomial identity. Therefore by [6, Theorem 2], $A \cap C \neq 0$ 
where $C=\operatorname{cent}(R)$. Suppose $c \in A \cap C$ and $c \neq 0$. Then $\left(c+c^{*}\right),\left(c c^{*}\right)$ are both in $A \cap C_{0}$. We claim either $c+c^{*} \neq 0$ or $c c^{*} \neq 0$. For if $c+c^{*}=0$, then $c=-c^{*}$. If $c c^{*}=0$ also, then $c^{2}=0$, so $c^{2}=0$, so $c=0$ since $R$ is semiprime, a contradiction. Thus $A \cap C_{0} \neq 0$. Q.E.D.

Corollary 1. If $(R, *)$ is as in Theorem 1 and $C_{0}$ is a field, then $(R, *)$ is simple.

Proof. Immediate from the theorem.

Now we have all the pieces to prove the following strong analogue to Posner's theorem.

THEOREM 2. Let $(R, *)$ be prime and satisfy a polynomial identity. $(R, *)$ has a ring of central quotients $(S, *)$ which is simple, finite dimensional over its center, which is the quotient field of the center of $(R, *) .(R, *)$ and $(S, *)$ satisfy the same homogeneous polynomial identities, and the involutions given on $R$ and on $S$ are of the same kind. Finally, $S$ is both the left and right ring of quotients for $R$.

Proof. We have noted that the center $C_{0}$ of $(R, *)$ is an integral domain, if $(R, *)$ is prime. Thus, the set of regular elements $T$ of $C_{0}$ is merely $C_{0}-\{0\}$. From Lemma 2 , we have the existence of $(S, *)=\left(R_{T}, *\right)$, with center $\left(C_{0}\right)_{T}$, which is the quotient field of $C_{0}$. But then $(S, *)$ is simple by Corollary 1 . We have already seen that $(R, *)$ and $(S, *)$ satisfy the same homogeneous polynomial identities, and that the involutions given on $R$ and on $S$ are of the same kind. To prove the rest of the theorem, we need an easy result on the structure of $S$ (given in [4, p. 14, Example 1]): Either $S$ is simple or $S=S_{1} \oplus S_{2}$ and $S_{1}^{*}=S_{2}, S_{1}$ and $S_{2}$ simple.

Clearly if $S$ is simple we are done by [6, Corollary to Theorem 2], the strong version of Posner's theorem (without regard to involution). So let $S=S_{1} \oplus S_{2}$. Since $S$ satisfies a polynomial identity, so do $S_{1}$ and $S_{2}$. Therefore $S_{1}$ and $S_{2}$ are finite dimensional over their respective centers $F_{1}$ and $F_{2}$. If $F=$ center of $(S, *)$ we have $F_{1}=(1,0) F$ and $F_{2}=(0,1) F$. Then for $i=1,2$, it is clear that $S_{i}$ as an $F$-algebra is isomorphic to $S_{i}$ as an $F_{i}$ algebra, so $S$ is finite dimensional over $F$. Finally, let $r_{1}$ be (both left and right) regular in $R$. Clearly $r_{1}$ is then regular in $S$, so $(1,0) r_{1}$ is regular in $S_{1}$ and $(0,1) r_{1}$ is regular in $S_{2}$. Since $S_{1}$ and $S_{2}$ are both simple artinian, there are $y_{1} \in S_{1}, y_{2} \in S_{2}$, such that $(1,0)=(1,0) r_{1} y_{1}=r_{1}\left((1,0) y_{1}\right)=r_{1} y_{1}$ and $(0,1)=(0,1) r_{1} y_{2}=r_{1} y_{2}$. Thus, $1=(1,0)+(0,1)=r_{1}\left(y_{1}+y_{2}\right)$, so $r_{1}$ is invertible in $S$, which shows that $S$ is the ring of quotients for $R$. Q.E.D.

We now observe that these results for rings with involution generalize the corresponding results for rings in general (disregarding involutions). To see this, let $R^{0}$ be the opposite ring of $R[4, \mathrm{p} .13]$, i.e. $R^{0}$ has the same additive group structure as $R$, but the product $r_{1} \circ r_{2}$ in $R^{0}$ is defined 
by $r_{1} \circ r_{2}=r_{2} r_{1}$. Let $R^{\prime}=R \oplus R^{0}$. $R^{\prime}$ has the multiplication given by $\left(r_{1}, s_{1}\right)\left(r_{2}, s_{2}\right)=\left(r_{1} r_{2}, s_{2} s_{1}\right)$ and can be given the exchange involution $J$ defined by $(r, s)^{J}=(s, r)$. Then there is a canonical correspondence of ideals in $R$ with ideals in $\left(R^{\prime}, J\right)$ given by $A \leftrightarrow A \oplus A^{0}$, and the center of $\left(R^{\prime}, J\right)$ is $\{(c, c) \mid c \in C\}$, where $C$ is the center of $R$. The results we prove for rings with involution hold for $\left(R^{\prime}, J\right)$, which shows that the corresponding theorems hold for $R$. For example, we claim that Posner's theorem (as stated in [6, Corollary to Theorem 1]) is a consequence of Theorem 2. For suppose $R$ is a prime ring with polynomial identity. Then $\left(R^{\prime}, J\right)$ is prime with the same polynomial identity and its ring of central quotients $(S, J)$ is simple by Theorem 2 . Thus $(1,0) S$ is the simple ring of quotients of $R$, and is easily seen to be the ring of central quotients of $R$, immediately yielding the desired result.

Appendix. We consider now generalizations of the preceding results. So let $(R, *)$ be a semiprime ring with polynomial identity and with center $C_{0}$, and let $T$ be the set of regular elements of $C_{0}$. Theorem 2 says that if $(R, *)$ is prime then $R$ has a classical ring of quotients, which is in fact $R_{T}$, and $\left(R_{T}, *\right)$ is simple. One might therefore ask of semiprime $(R, *)$ :

(1) Is $R_{T}$ a ring of quotients for $R$ ?

(2) Is $\left(R_{T}, *\right)$ semisimple?

Neither conjecture is true in general. In fact, an interesting commutative counterexample to (2) is the following: Let $X_{1}, X_{2}, \cdots$ be an infinite set of indeterminates, and set $X_{i} X_{j}=0$ for $i \neq j$. For any field $F$, let $R=$ $F\left[\left[X_{1}, X_{2}, \cdots\right]\right]$ be the ring of formal power series, each element using only a finite number of indeterminates. A typical element is

$$
r=\alpha_{0}+\sum_{i \geqq 1} \alpha_{1 i} X_{1}^{i}+\sum_{i \geqq 1} \alpha_{2 i} X_{2}^{i}+\cdots+\sum_{i \geqq 1} \alpha_{m i} X_{m}^{i}
$$

where $m<\infty$.

It is easy to see that $r$ is regular if and only if $\alpha_{0} \neq 0$, in which case $r$ also has an inverse in $R$. Thus $R$ is its own ring of central quotients. Although $R$ is semiprime, $R$ is certainly not semisimple since it has only one maximal ideal (the set of power series with constant term 0 ). However, there is a decent theorem which generalizes Theorem 2 for certain semiprime $(R, *)$; with polynomial identity, namely:

Suppose that among the prime ideals of $(R, *)$ there is a finite set whose intersection is 0 . Then $R_{T}$ is semisimple artinian and is the ring of quotients for $R$.

A modification of an argument of Herstein given in Lemma 11 of [3, p. 269] shows that the hypothesis of the above theorem is satisfied if $(R, *)$ satisfies the following conditions:

(i) Every set of independent ideals of $(R, *)$ is finite. 
(ii) Every set of left annihilators of ideals $(R, *)$ contains a maximal element. (In the situation under consideration, (ii) implies (i).)

Actually the left annihilator of any ideal $A$ of $(R, *)$ is also an ideal of $(R, *)$. For let Ann $A$ be the left annihilator of $A$. Clearly Ann $A$ is an ideal of $R$. Moreover, $A(\operatorname{Ann} A)^{*}=\left((\operatorname{Ann} A) A^{*}\right)^{*}=((\operatorname{Ann} A) A)^{*}=0$. But then $\left((\operatorname{Ann} A)^{*} A\right)^{2}=0$, and since $R$ is semiprime (Ann $\left.A\right)^{*} A=0$. Thus, (Ann $A)^{*} \subseteq$ Ann $A$, which implies Ann $A$ is an ideal of $(R, *$ ).

Conditions (i) and (ii) generalize slightly the Goldie conditions of [3, p. 263], so we shall call a ring $(R, *)$ which satisfy these conditions a "quasi-Goldie" ring. Thus, for any quasi-Goldie semiprime $(R, *)$ with polynomial identity, its ring of central quotients is the ring of (both left and right) quotients, which is semisimple artinian.

Details of all of the above assertions will be given in the author's forthcoming dissertation.

\section{REFERENCES}

1. S. A. Amitsur, Prime rings having polynomial identities, Proc. London Math. Soc. (3) 17 (1967), 470-486. MR 36 \#209.

2. — Identities in rings with involutions, Israel J. Math. 7 (1969), 63-68. MR 39 \#4216.

3. N. Jacobson, Structure of rings, rev. ed., Amer. Math. Soc. Colloq. Publ., vol. 37, Amer. Math. Soc., Providence, R.I., 1964. Chapter X and Appendix B. MR 36 \#5158.

4. - - Structure and representation of Jordan algebras, Amer. Math. Soc. Colloq. Publ., vol. 39, Amer. Math. Soc., Providence, R.I., 1968. Sections I.4 and V.7. MR 40 \#4330.

5. W. S. Martindale III, Rings with involution and polynomial identities, J. Algebra 11 (1969), 186-194. MR 38 \#3302.

6. L. H. Rowen, Some results on the center of a ring with polynomial identity, Bull. Amer. Math. Soc. 79 (1973), 219-223.

Department of Mathematics, Yale University, New Haven, Connecticut 06520

Current address: Department of Mathematics, University of Chicago, Chicago, Illinois 60637 